\title{
Brain MRI in a patient with classical galactosemia: acute event of unilateral hemispheric cerebral edema
}

\author{
Tiago Gil Oliveira ${ }^{1,2,3}$ (D) João Paulo Soares-Fernandes ${ }^{1}$
}

Received: 28 August 2017 / Accepted: 1 September 2017 / Published online: 12 September 2017

(C) Springer-Verlag GmbH Germany 2017

Dear editor,

Classical galactosemia or galactosemia type 1 is caused by galactose-1-phosphate uridylyltransferase deficiency and characterized by the toxic accumulation of galactose-1-phosphate and galactitol [2]. Early-life clinical manifestations include failure to thrive, vomiting, diarrhea, hepatomegaly, and jaundice. Although galactose dietary restriction prevents most of these symptoms, in the long-term, the central nervous system is still affected. Decreased verbal and cognitive performance, tremor, ataxia, and seizures are possible manifestations [2]. On imaging, generalized atrophy with cerebellar

Tiago Gil Oliveira

tiago@med.uminho.pt

Division of Neuroradiology, Hospital de Braga, Braga, Portugal

2 Life and Health Sciences Research Institute (ICVS), School of Medicine, University of Minho, Campus Gualtar, 4710-057 Braga, Portugal

3 ICVS/3B's_-PT Government Associate Laboratory, Braga, Guimarães, Portugal predominance and abnormal myelination with multiple white matter high-intensity foci have been reported [4]. In the untreated stages of the disease, proton magnetic resonance spectroscopy (MRS) may depict an abnormal peak representing galactitol associated with white matter edema [1].

A 10-year-old female with classical galactosemia, followed on out-patient clinic and on galactose-restricted diet, presented with an acute event characterized by decreased level of consciousness and left hemiparesis. Magnetic resonance imaging (MRI) revealed right hemisphere vasogenic edema, causing sulcal effacement and midline shift (Fig. 1a-d). Even though galactitol was identified through MRS (Fig. 1g, h) coexistent with edema, the absent restricted diffusion (Fig. 1c, d) and anisotropy preservation (Fig. 1e, f) suggest a potentially reversible lesion. The patient recovered from this acute event with no focal neurological deficits. We present here characteristic MRI findings with galactitol accumulation with associated edema in an unusual unilateral pattern. Since galactose dietary restrictions did not show consistently a more favorable outcome [3], this case emphasizes the role of multimodal MRI techniques in evaluating acute and chronic brain damage caused by galactosemia, and, likely, in monitoring therapeutic efficacy. 

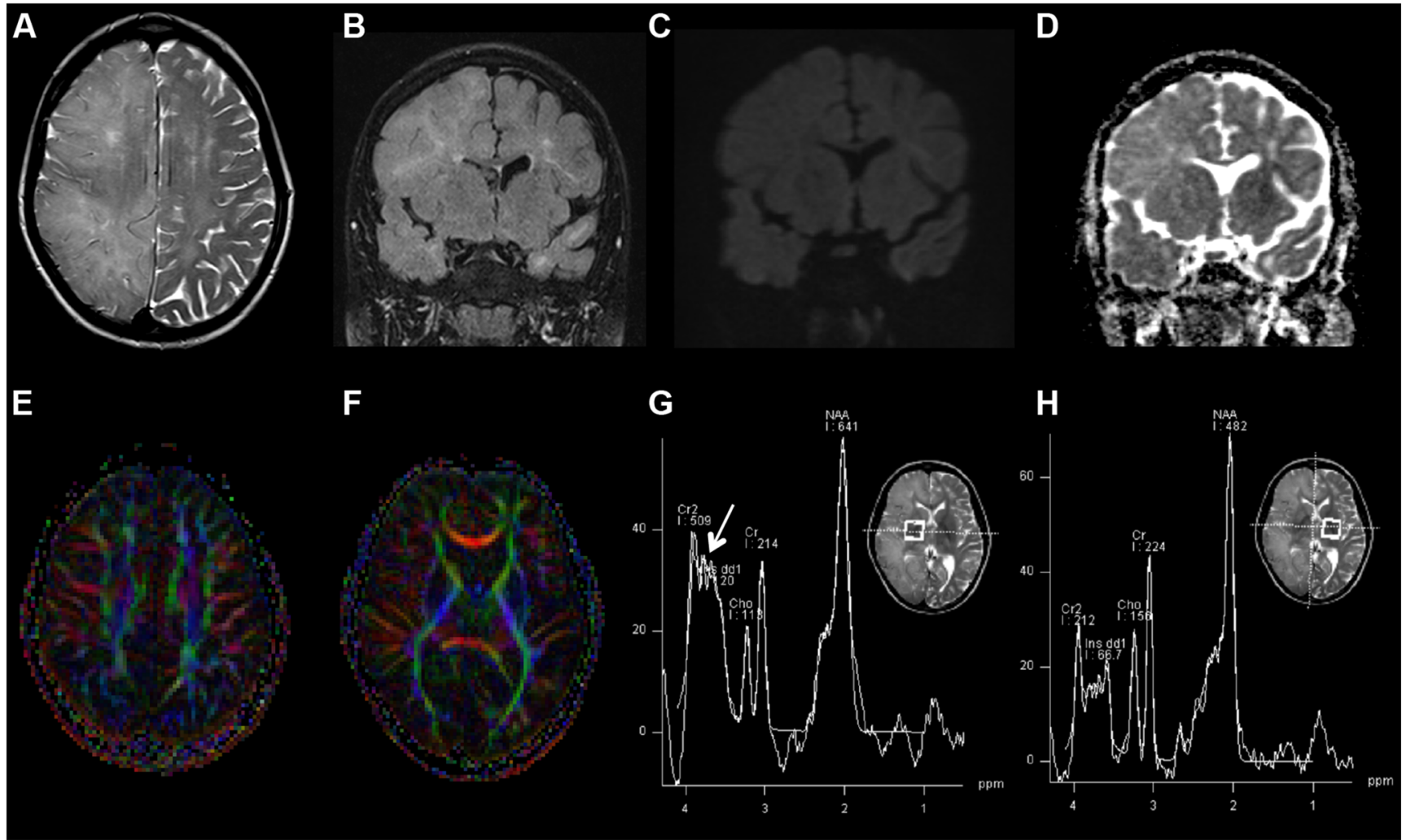

Fig. 1 Magnetic resonance imaging (MRI) of a 10-year-old female with the diagnosis of galactosemia, in the context of a new episode of acute complication with decreased consciousness and left hemiparesis. Axial T2 image (a) and coronal fluid-attenuated inversion recovery (FLAIR) images (b) reveal pathologic high signal intensity and sulcal effacement in the right hemisphere; previous changes of gliotic foci, brain atrophy, and overall hypomyelination remain in the contralateral hemisphere. Coronal diffusion-weighted image (DWI, $b=1000 \mathrm{~mm}^{2} / \mathrm{s}$ ) (c) and apparent diffusion coefficient (ADC) map (d) show corresponding

\section{Compliance with ethical guidelines}

Conflict of interest On behalf of all authors, the corresponding author states that there is no conflict of interest.

\section{References}

1. Berry GT, Hunter JV, Wang Z, Dreha S, Mazur A, Brooks DG, Ning C, Zimmerman RA, Segal S (2001) In vivo evidence of brain galactitol accumulation in an infant with galactosemia and slight increase in water diffusion, suggesting vasogenic edema. Axial color-coded diffusion tensor imaging (DTI) of the semioval center (e) and basal ganglia levels (f) shows preserved anisotropy in white matter tracts. MR spectroscopy, TE $=30 \mathrm{~ms}$, ROI on the right basal ganglia (g) shows the abnormal presence of a galactitol double peak at 3.6 and $3.7 \mathrm{ppm}$ (white arrow). Galactitol to creatine ratio is approximately 1 . On the left side (h), a much less conspicuous galactitol peak is seen; galactitol to creatine ratio is $<0.5$. $(\mathrm{Cr})$ creatine; (Cho) choline; (NAA) $\mathrm{N}$-acetylaspartate

encephalopathy. J Pediatr 138:260-262. https://doi.org/10.1067/ mpd.2001.110423

2. Bosch AM (2006) Classical galactosaemia revisited. J Inherit Metab Dis 29:516-525. https://doi.org/10.1007/s10545-006-0382-0

3. Hughes J, Ryan S, Lambert D, Geoghegan O, Clark A, Rogers Y, Hendroff U, Monavari A, Twomey E, Treacy EP (2009) Outcomes of siblings with classical galactosemia. J Pediatr 154:721-726. https://doi.org/10.1016/j.jpeds.2008.11.052

4. Nelson MD Jr, Wolff JA, Cross CA, Donnell GN, Kaufman FR (1992) Galactosemia: evaluation with MR imaging. Radiology 184:255-261. https://doi.org/10.1148/radiology.184.1.1319076 